\title{
Exact solutions for discs around stationary black holes
}

\author{
N. Požár, O. Semerák, J. Šácha, M. Žáček and T. Zellerin \\ Inst. of Theor. Physics, Faculty of Maths and Phys., Charles Univ., Prague, Czech Republic \\ email: oldrich.semerak@mff.cuni.cz
}

\begin{abstract}
Black holes surrounded by axisymmetric structures prosper in some of the most interesting sources in the universe. However, a consistent exact description of the gravitational field of these systems is still lacking. In a static case, the task reduces to Laplace equation and the fields of multiple sources follow by mere superposition. In a rotating case, non-linearity of the Einstein equations resists simple grasp, but even then the theory of completely integrable systems seems to verge on satisfactory solutions. It seeks them in terms of $\theta$-functions on special manifolds connected - symptomatically - with the names of Riemann and Hilbert.
\end{abstract}

Keywords. Accretion disks - black hole physics - gravitation - relativity

All the evidence for black holes is based on their interaction with the surrounding matter, but in theoretical models the gravity of this matter is neglected: the field is fully due to the hole as given by general relativity. Certainly, real accretion discs are probably much lighter than their central holes, but (i) this is not always the case (black hole with neutron torus as a temporary stage of a compact-binary collapse), and (ii) some important properties of the disc flow were found sensitive to the details of the gravitational field. However, general relativity being non-linear, it is not simple to incorporate the disc effect.

Einstein equations can be treated numerically and for light additional sources a perturbative approach may also be adequate, but only an exact solution can convey all the richness of the theory with full accuracy and generality. In the case of stationarity and axial symmetry, the field outside of sources can be described by the metric

$$
\mathrm{d} s^{2}=-f(\mathrm{~d} t+A \mathrm{~d} \phi)^{2}+f^{-1}\left[\rho^{2} \mathrm{~d} \phi^{2}+e^{2 k}\left(\mathrm{~d} \rho^{2}+\mathrm{d} z^{2}\right)\right]
$$

in the Weyl-Lewis-Papapetrou cylindrical coordinates $(\rho, \phi, z)$. The unknown functions $f(\rho, z), A(\rho, z)$ must satisfy the Ernst equation $f \vec{\nabla}^{2} \mathcal{E}=(\vec{\nabla} \mathcal{E})^{2}$ for the complex (Ernst) potential $\mathcal{E} \equiv f+\mathrm{i} \psi$ with $\psi$ introduced by $\rho \psi_{, z}=f^{2} A_{, \rho}, \rho \psi_{, \rho}=-f^{2} A_{, z}$; index-posed commas denote partial derivatives, $\vec{\nabla} \equiv\left(\partial_{\rho}, \partial_{z}\right)$ and $\vec{\nabla}^{2}=\partial_{\rho \rho}+\frac{1}{\rho} \partial_{\rho}+\partial_{z z}$.

Up to date, multi-body exact solutions have only been studied in more detail in a static case $(A=0)$ when the equation reduces to Laplace equation for $f$ and compound fields follow by linear superposition plus a line integration for $k(\rho, z)$. In particular, we explored the Schwarzschild black hole surrounded, in the equatorial plane, by several types of sources - thin discs (i) of the inverted Morgan-Morgan family (Lemos \& Letelier 1994; Semerák et al. 1999; Semerák \& Žáček 2000; Žáček \& Semerák 2002; Letelier 2003; Semerák 2003) and (ii) of the inverted family with power-law density profiles (Semerák 2004), (iii) Bach-Weyl thin ring (Chakrabarti 1988; Semerák et al. 1999), and (iv) thick toroid (Š́cha \& Semerák 2005). The total fields were e.g. studied on field lines, geodesics, equatorial circular motion or redshift behaviour. We observed that

- the disc's gravity affects Keplerian motion (in terms of which the disc itself is interpreted), the density profile being the most important "input"; the real accretion discs may thus be sensitive to their own field, even if their mass is not big relative to the centre 
- this mainly applies to the disc's stability; for some density profiles, instability first occurs inside the disc (not at the rim) when its rim is shifted closer and closer to the hole, which "in practice" could imply radial fragmentation of the disc.

Such "ad-hoc" static superpositions are far from describing real systems: they are not linked with any accretion model and do not involve rotation. However, some of them possess physically acceptable properties within a reasonable range of parameters, so they can at least indicate some effects that might occur. (See Karas et al. 2004 for a review.)

In stationary setting, the problem gets fully non-linear and much more involved. Wide classes of new generic solutions have still been found - by "generating techniques" developed in 1950s-1980s. Unfortunately, it is almost impossible to impose physical prerequirements on these mathematical procedures and most of their outcomes have hardly any relevance. We made just minor attempt in this direction (Zellerin \& Semerák 2000), to derive a solution for a rotating hole with a thin disc by a certain version of the "inverse-scattering method". The result was not physical (Semerák 2002).

The "generating" algorithms translate the original, non-linear equations into a pair of linear equations. In the last decade, the linear problem equivalent to the Ernst equation has been addressed using the ideas of algebraic geometry (e.g. Ansorg et al. 2002; Korotkin 2004; Klein \& Richter 2005; and references therein). Instead of the boundaryvalue problem for $\mathcal{E}$, the Riemann-Hilbert problem is tackled to find certain functions having prescribed jump(s) across prescribed contour(s) on a specific Riemann surface. The solution is not known in general, but in some cases it can be given in terms of Riemann $\theta$ functions. The method seems to verge on providing results that can have physical relevance (Frauendiener \& Klein 2001; Klein 2003); in particular, numerical codes were created for evaluation of theta functions (Frauendiener \& Klein 2004; Frauendiener \& Klein 2006; Požár 2006). Now it should mainly be clarified what properties of the Riemann surfaces correspond to particular spacetime features and locations in order that the scheme might be supplemented by a detailed and running physical control.

We thank V. Karas and colleagues from the Prague Relativity Group.

\section{References}

Ansorg, M., Kleinwächter, A., Meinel, R. \& Neugebauer, G. 2002, Phys. Rev. D, 65, 044006

Chakrabarti, S. K. 1988, J. Astrophys. Astron., 9, 49

Frauendiener, J. \& Klein, C. 2001, Phys. Rev. D, 63, 084025

Frauendiener, J. \& Klein, C. 2004, J. Comp. Appl. Math., 167, 193

Frauendiener, J. \& Klein, C. 2006, Lett. Math. Phys., 76, 249

Karas, V., Huré, J.-M. \& Semerák, O. 2004, Class. Quantum Grav., 21, R1

Klein, C. 2003, Phys. Rev. D, 68, 027501

Klein, C. \& Richter, O. 2005, Ernst equation on hyperelliptic Riemann surfaces (Springer, Berlin)

Korotkin, D. A. 2004, Math. Ann., 329, 335

Lemos, J. P. S. \& Leterier, P. S. 1994, Phys. Rev. D, 49, 5135

Letelier, P. S. 2003, Phys. Rev. D, 68, 104002

Požár, N. 2006, diploma thesis (Charles Univ., Faculty Math. \& Phys., Prague)

Šácha, J. \& Semerák, O. 2005, Czech. J. Phys., 55, 139

Semerák, O. 2002, Class. Quantum Grav., 19, 3829

Semerák, O. 2003, Class. Quantum Grav, 20, 1613

Semerák, O. 2004, Class. Quantum Grav., 21, 2203

Semerák, O. \& Žáček, M. 2000, Class. Quantum Grav., 17, 1613

Semerák, O., Zellerin, T. \& Žáček, M. 1999, MNRAS, 308, 691 (Erratum: 2001322 207) and 705

Žáček, M. \& Semerák, O. 2002, Czech. J. Phys., 52, 19

Zellerin, T. \& Semerák, O. 2000, Class. Quantum Grav., 17, 5103 